\title{
Lung microbiota and COVID-19 severity
}

\author{
Direct sampling of lung alveoli of critically-ill patients infected with SARS-CoV-2 shows that lung microbiota and an \\ impaired alveolar immune response together are predictive of poor clinical outcomes.
}

\author{
Robert P. Dickson
}

(

ne of the more confounding aspects of the SARS-CoV-2 pandemic has been the unpredictable heterogeneity of the clinical manifestations of viral infection. The same viral strain, infecting demographically similar hosts, can provoke a tremendous range of disease severity, from asymptomatic carriage to life-threatening respiratory failure. In this issue of Nature Microbiology, Sulaiman et al. reveal the importance of directly sampling SARS-CoV-2-infected lungs to understand the microbiology and heterogeneity of COVID-19. The authors sampled the lower respiratory tract of critically ill patients with COVID-19 using bronchoscopy - a regrettably uncommon approach in the study of COVID-19 - to discover that site-specific microbiologic and immunologic features of the lower respiratory tract explain variation in both viral load and clinical outcomes. These new findings suggest that one of the major blind spots in the field's approach to understanding SARS-CoV-2 pathogenesis has been our failure to directly sample and study the alveolar space.

Clinical heterogeneity arising from SARS-CoV-2 infection has been central to the success of this coronavirus as a pandemic pathogen. On the one hand, if SARS-CoV-2 infection were more uniformly lethal, it would probably be easier to contain because severely ill patients tend to self-isolate, and deceased patients are effectively non-communicable. On the other hand, if infection were more uniformly mild, SARS-CoV-2 would be just another seasonal 'common cold' coronavirus. The rapid spread of SARS-CoV-2 and its persistence worldwide are due in part to its seemingly stochastic ability to infect most hosts with asymptomatic or mild disease (ensuring widespread transmission) while condemning an unlucky minority to lethal lung injury.

As we approach two years of intense study of SARS-CoV-2, the clinical heterogeneity arising from virus infection still cannot be completely explained. At a coarse epidemiological level, older age, comorbidities and low socioeconomic status

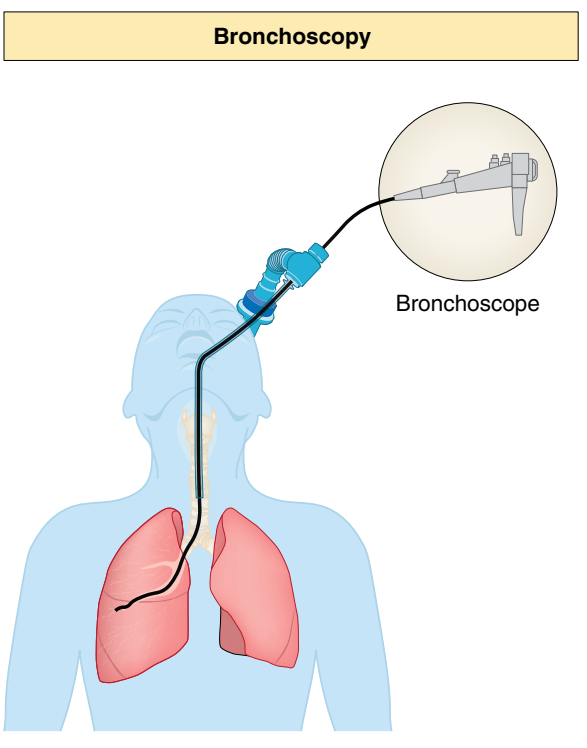

Fig. 1 | The overlooked importance of directly sampling the lungs in SARS-CoV-2 infection.

The standard method used to sample the lower respiratory tract in humans is bronchoscopy, in which the alveolar space can be sampled by saline lavage. Due to concerns regarding aerosolization and transmission risk, bronchoscopy has been avoided for patients with COVID-19, and surrogate measurements (for example, from blood and pharyngeal swabs) have been used instead. The study by Sulaiman et al. reveals the inadequacy of this approach. In critically ill patients with COVID-19, site-specific microbiologic and immunologic features of the lower respiratory tract explain variation in viral load and clinical outcomes.

contribute to an increased risk of severe disease or death. Genome-wide association studies have identified several genetic loci that are associated with severe disease ${ }^{2}$, and immunophenotyping of peripheral leukocytes has revealed immunologic variation with plausible prognostic and pathophysiological significance ${ }^{3}$. However, patient-level variation in COVID-19 clinical severity remains largely unexplained.

Severe COVID-19 is, above all, a viral pneumonia that provokes epithelial and endothelial injury in the lower respiratory tract. Unfortunately, sampling the alveolar space, which is the primary site of disease pathogenesis, is challenging. For decades, the most useful and informative approach (used in the study by Sulaiman et al.) has been to use bronchoscopy. This involves inserting a thin, flexible tube through the larynx into the lower respiratory tract, where it can be used to sample the small airways (via brushings) and alveolar space (via bronchoalveolar lavage) (Fig. 1). Early in the SARS-CoV-2 pandemic, most clinical centres and research investigators elected to avoid bronchoscopy in patients with COVID-19, owing to concerns about virus aerosolization and the ensuing increased risk of transmission. Consequently, nearly all human studies to date on the biology of SARS-CoV-2 infection have not sampled the lungs. The literature has instead been dominated by studies using blood, pharyngeal swabs and other more conveniently acquired specimen types.

In retrospect, our decision to forego bronchoscopic sampling in the study of COVID-19 was a mistake. First, we now know that when appropriate precautions and personal protective equipment are used, the risk of transmission to bronchoscopists and support staff is exceedingly low ${ }^{4}$. Second, in terms of biological relevance, we already knew from pre-COVID-19 studies that in severe lung injury, alveolar inflammation is barely (if at all) correlated with the systemic indices of inflammation that are measured in peripheral blood ${ }^{5,6}$. What is immunologically, microbiologically or pathologically occurring in the alveoli cannot be inferred by studying the blood. In the COVID-19 era, over-interpretation of studies using surrogate measurements has clouded our understanding of disease pathogenesis. Examples include the purported 'cytokine storm', the overstated clinical importance of pulmonary microthrombosis ${ }^{8}$, and the widely disseminated but exhaustively refuted 'subphenotypes' of severe COVID-19 derived from premature and pathophysiological inferences derived from premature and 
underpowered physiologic observations ${ }^{9}$. Finally, the few studies to date that have characterized bronchoscopic specimens from patients with COVID-19 have proven transformative in their illumination of SARS-CoV-2 pathogenesis ${ }^{10}$.

In their study, Sulaiman et al. carried out bronchoalveolar lavage on 142 patients infected with SARS-CoV-2 and used complementary molecular techniques to study both the microbiota (bacterial and viral) of the lower respiratory tract and the host immune response. By leveraging these methodologically robust and anatomically relevant measurements and collecting rigorously adjudicated clinical outcome data, the authors identified new molecular predictors of poor clinical outcomes (death or prolonged mechanical ventilation $)^{1}$.

The authors found that both the total burden and taxonomic identity of lower respiratory tract bacteria (characterized molecularly using bronchoalveolar lavage specimens) were independently predictive of death and prolonged mechanical ventilation. This new finding validates prior observations for other causes of severe lung injury ${ }^{11}$ and would have been unappreciated using either conventional culture-based techniques or pharyngeal swabs: culture-detected bacterial co-infection was not predictive of poor outcomes in this cohort, and time-matched upper respiratory tract communities contained bacterial community signatures distinct from those of alveolar specimens. While several studies have attempted to correlate pharyngeal and rectal microbiota with clinical features of COVID-19, the current findings support prior work suggesting that lung microbiota play a more immediate role in calibrating alveolar immunity than oral or lower gut microbiota ${ }^{12}$.

Similarly, the authors discovered that the prognostic significance of SARS-CoV-2 viral load (a topic of considerable controversy) depends entirely on the anatomic site of sampling. While viral load in alveolar specimens was a convincing predictor of mortality, the same was not true for time-matched upper respiratory tract specimens. While the viral load of paired upper and lower respiratory tract specimens was correlated across patients, the difference in prognostic significance across anatomic sites affirms both the site-specificity of viral replication in critically ill patients with COVID-19 as well as the limitations of relying on surrogate samples.

Finally, by characterizing the host response in bronchoalveolar lavage specimens, the authors showed that alveolar concentrations of SARS-CoV-2 antibodies (anti-spike and anti-RBD) were lower in patients who died and were negatively correlated with viral replication (as quantified using sub-genomic RNA). Similarly, poor clinical outcomes were predicted by differences in alveolar gene expression, including downregulation of genes necessary for immunoglobulin ( $\operatorname{IgA}$ and $\operatorname{IgG}$ ) production. Thus, contrary to the widespread (and conceptually fraught) belief that severe lung injury in COVID-19 is attributable to an inappropriately exuberant immune response ${ }^{7}$, the authors provide molecular and site-specific (alveolar) evidence that virus-specific immune deficiency plays a role in progressive, non-resolving lung injury.

Taken together, the findings of Sulaiman et al. shed considerable light on the dark matter of COVID-19 heterogeneity. Variation in clinical outcomes is partly explained by alveolar viral burden, lung microbial communities and an inadequate adaptive alveolar immune response. Beyond the immediate research questions suggested by these findings (for example, whether these microbiological and immunologic alveolar signatures represent 'treatable traits' in severe COVID-19, whether bacterial burden and viral load in the alveolar space are viable therapeutic targets in critically ill patients with COVID-19, and whether the alveolar anti-SARS-CoV-2 immunoglobulin response can be therapeutically augmented), these findings also remind us that if we aspire to understand the biology of severe lung infections like COVID-19, there is simply no substitute for studying the lungs themselves.

\section{Robert P. Dickson (D) 1,2,3凶}

${ }^{1}$ Division of Pulmonary and Critical Care Medicine, Department of Internal Medicine, University of Michigan Medical School, Ann Arbor, MI, USA. ${ }^{2}$ Department of Microbiology and Immunology, University of Michigan Medical School, Ann Arbor, MI, USA. ${ }^{3}$ Michigan Center for Integrative Research in Critical Care, Ann Arbor, MI, USA.

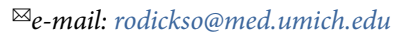

Published online: 27 September 2021

https://doi.org/10.1038/s41564-021-00969-X

References

1. Sulaiman, I. et al. Nat. Microbiol. https://doi.org/10.1038/s41564021-00961-5 (2021).

2. COVID-19 Host Genetics Initiative. Nature https://doi.org/ 10.1038/s41586-021-03767-x (2021).

3. Mathew, D. et al. Science https://doi.org/10.1126/science.abc8511 (2020).

4. Gao, C. A. et al. Ann. Am. Thor. Soc. 18, 1243-1246 (2021).

5. Morrell, E. D. et al. Am. J. Respir. Crit. Care Med. 197, 528-532 (2018).

6. Heijnen, N. F. L. et al. Physiol. Rep. 9, el4693 (2021).

7. Sinha, P., Matthay, M. A. \& Calfee, C. S. JAMA Intern. Med. 180, 1152-1154 (2020).

8. The REMAP-CAP, ACTIV-4a \& ATTACC Investigators. N. Engl. J. Med. https://doi.org/10.1056/NEJMoa2103417 (2021).

9. Bos, L. D. J., Sinha, P. \& Dickson, R. P. Eur. Respir. J. https://doi. org/10.1183/13993003.01768-2020 (2020).

10. Grant, R. A. et al. Nature 590, 635-641 (2021).

11. Dickson, R. P. et al. Am. J. Respir. Crit. Care Med. 201, 555-563 (2020).

12. Dickson, R. P. et al. Am. J. Respir. Crit. Care Med. 198, 497-508 (2018).

Competing interests

R.P.D. has received funding from the NIH (R01HL144599 and T32HL007749). 